\title{
Optimal Integration Time for UWB Transmitted Reference Correlation Receivers
}

\author{
Yi-Ling Chao \\ Communication Sciences Institute \\ University of Southern California, Los Angeles, California 90089-2565 \\ Email: yilingch@usc.edu
}

\begin{abstract}
This paper derives the bit error probability (BEP) of the conventional and average correlation receivers of the ultrawideband transmitted reference system by exploiting the concept of the orthogonal expansion, and the central limit theorem is not needed in the derivation. The effects of using different lengths of the integration time in the conventional correlation receiver in a single user multipath environment are also discussed. Three optimization criteria are evaluated, and the average BEPs adopted the optimal integration time based on these criteria do not differ significantly from each other. In the interested BEP range, about $2 \mathrm{~dB}$ can be gained by using the optimal integration time instead of the channel delay spread in the correlator. ${ }^{1}$
\end{abstract}

\section{INTRODUCTION}

Ultra-wideband (UWB) impulse radio systems transmit data by modulation of subnanosecond pulses. These narrow pulses are distorted by the channel, but often can resolve many distinct propagation paths (multipath) because of their fine time-resolution capability [1]. However, a Rake receiver that implements tens or even hundreds of correlation operations may be required to take full advantage of the available signal energy [2]. On the other hand, a receiver using a single correlator matched to one transmission path may be operating at a $10-15 \mathrm{~dB}$ signal energy disadvantage relative to a full Rake receiver.

A Transmitted reference scheme, which can ease the stringent receiver requirement of the synchronization, channel estimation, and a Rake reception has attracted lots of attention [3]. In this TR modulated system, a reference waveform is transmitted before each data-modulated waveform for the purpose of determining the current multipath channel response. The proposed conventional correlation receiver correlates the data signal with the reference signal to acquire all the energy without requiring additional channel estimation and Rake reception. One major drawback of this simple receiver structure is the transmitted reference signal used as a correlator template is noisy. One method to clean the correlator template is to use the average correlation receiver, which average reference waveforms in one bit duration as the template with the penalty of increasing the receiver complexity. The bit error probabilities (BEPs) of these two receiver were evaluated with the help of the central limit theorem [3], [4]. This evaluation, although an approximation, can assess the BEPs accurately when the noise bandwidth $\times$ time dimension is large enough

\footnotetext{
${ }^{1}$ This work was supported by the Army Research Office under MURI Grant No. DAAD19-01-1-0477.
}

which is usually the case in a UWB system. The BEPs of correlation receivers with an ideal front-end bandpass filter are evaluated in this paper by exploiting the concept of the orthogonal expansion in section II.

The original idea of the conventional receiver is to capture all the energy available in a UWB multipath channel. But for the BEP performance, the correlator adopting the channel delay spread as the integration time is not the best choice. The received signal energy is small in the tail of the channel response, and the receiver can get more noise power than the signal energy through the excessive integration. This paper optimizes the integration time under three criteria which are minimizing the average BEP, maximizing the average decision signal energy to the noise power ratio (SNR), and maximizing the average decision SNR in which the received signal energy is replaced by its mean value (Section III). The results of these three criteria are compared in Section IV. Section V draws the conclusion.

\section{UWB TR MODUlation AND CORRELATION RECEIVERS}

The transmitted signal of a conventional UWB TR system with antipodal modulation is

$$
s_{\mathrm{tr}}(t)=\sum_{i=-\infty}^{\infty} g_{\mathrm{tr}}\left(t-i T_{\mathrm{f}}\right)+b_{\left\lfloor i / N_{\mathrm{s}}\right\rfloor} g_{\mathrm{tr}}\left(t-i T_{\mathrm{f}}-T_{\mathrm{d}}\right) .
$$

Here $g_{\mathrm{tr}}(t)$ is a transmitted monocycle waveform that is non-zero only for $t \in\left(0, T_{\mathrm{p}}\right)$, and $T_{\mathrm{f}}$ is the frame time. Each frame contains two monocycle waveforms. The first is a reference and the second, $T_{\mathrm{d}}$ seconds later, is a datamodulated waveform. The data bits $b_{\left\lfloor i / N_{s}\right\rfloor} \in\{1,-1\}$ with equal probability, and $\left\lfloor i / N_{\mathrm{s}}\right\rfloor$ is the integer part of $i / N_{\mathrm{s}}$. Hence each bit is transmitted in $N_{\mathrm{s}}$ successive frames to achieve an adequate bit energy in the receiver, and the channel is assumed invariant over this bit time.

In this TR system, $T_{\mathrm{d}}$ is greater than the multipath delay spread $T_{\mathrm{mds}}$ to assure that no interference between reference signal and data signal exists. The frame time is designated to be $T_{\mathrm{f}}=2 T_{\mathrm{d}}>2 T_{\mathrm{mds}}$ so that no interframe interference exists. Because the single user case is considered here, the time-hopping and/or direct sequence modulation which is used to reduce multiuser interference is eliminated for simplicity, but without loss of generality. 
We model the received TR signal $r(t)$ of bit $b_{0}$ in a stationary channel over a bit time by

$$
r(t)=\sum_{i=0}^{N_{\mathrm{s}}-1}\left[g\left(t-i T_{\mathrm{f}}\right)+b_{\left\lfloor i / N_{\mathrm{s}}\right\rfloor} g\left(t-i T_{\mathrm{f}}-T_{\mathrm{d}}\right)\right]+n(u, t),
$$

where $n(u, t)$ represents band-limited white Gaussian receiver noise with one-sided power spectral density $N_{0}$ and bandwidth $B_{\mathrm{w}}$, and $g(t)$ is the received waveform which is the convolution of a transmitted monocycle waveform $g_{\mathrm{tr}}(t)$ and channel impulse responses including effects of antennas and the front-end bandpass filter. A conventional correlation receiver correlates the received data-modulated waveform with the reference waveform, which is received $T_{\mathrm{d}}$ seconds earlier, and sums the $N_{\mathrm{s}}$ correlator outputs that are affected by a single data bit to be the decision statistic. The decision rule of bit $b_{0}$ is

$$
D_{\mathrm{s}}=\sum_{j=0}^{N_{\mathrm{s}}-1} \int_{j T_{\mathrm{f}}+T_{\mathrm{d}}}^{j T_{\mathrm{f}}+T_{\mathrm{d}}+T_{\text {corr }}} r\left(t-T_{\mathrm{d}}\right) r(t) d t \underset{-1}{\stackrel{1}{\gtrless}} 0,
$$

where $T_{\text {corr }} \leq T_{\mathrm{mds}}$ is the correlator's integration time.

With an ideal bandpass filter, the BEP can be evaluated by applying the orthogonal expansion technique as well as Appendix 9A in [5]. Without actually implementing it in the conventional receiver, we use the concept that both the bandpass signal and noise have complex lowpass equivalence. The energy in the lowpass equivalence of the received waveform $\hat{g}(t)$ is twice the energy in $g(t)$. The lowpass equivalence of the bandlimited noise $\hat{n}(u, t)$ has power spectral density $2 N_{0}$ from $-B_{\mathrm{w}} / 2$ to $B_{\mathrm{w}} / 2$, and zero elsewhere. In addition to the lowpass equivalence, another useful theorem states that a timelimited ( $\left.T_{\text {corr }}\right)$ band-limited $\left(B_{\mathrm{w}}\right)$ signal has dimension $B_{\mathrm{w}} T_{\text {corr }}$ at most, and can be represented by $\left\{b_{k}(t)\right\}_{k=1}^{B_{\mathrm{w}} T_{\text {corr }}}$, a complete orthonormal set with

$$
\int_{0}^{T_{\mathrm{corr}}} b_{j}(t) b_{k}^{*}(t) d t=\left\{\begin{array}{cc}
0 & j \neq k \\
1 & j=k
\end{array}\right.
$$

where $b_{k}^{*}(t)$ is the complex conjugate of $b_{k}(t)$. Thus the lowpass equivalence of the filtered signal and noise with time duration $T_{\text {corr }}$ can be represented by the complete set $\left\{b_{k}(t)\right\}_{k=1}^{B_{\mathrm{w}} T_{\text {corr }}}$. The complex lowpass equivalence of the received waveform is now written as

$$
\hat{g}(t)=\sum_{k=1}^{B_{\mathrm{w}} T_{\text {corr }}} \hat{g}_{k} b_{k}(t)
$$

where the weights are

$$
\hat{g}_{k}=\int_{0}^{T_{\text {corr }}} \hat{g}(t) b_{k}^{*}(t) d t,
$$

and

$$
\eta E_{\mathrm{p}}=\int_{0}^{T_{\text {corr }}} g^{2}(t) d t=\frac{1}{2} \sum_{k=1}^{B_{\mathrm{w}} T_{\text {corr }}} \hat{g}_{k}^{2}
$$

with $E_{\mathrm{p}}=\int_{0}^{T_{\mathrm{mds}}} g^{2}(t) d t$ being the total energy in a receiver waveform, and $\eta$ being the efficiency factor. The noise covariance function of $\hat{n}(u, t), K_{\hat{n} \hat{n}^{*}}\left(t_{1}, t_{2}\right)$, satisfies

$$
2 N_{0} b_{k}\left(t_{1}\right)=\int_{0}^{T_{\text {corr }}} K_{\hat{n} \hat{n}^{*}}\left(t_{1}, t_{2}\right) b_{k}\left(t_{2}\right) d t_{2}
$$

for $k=1,2, \ldots, B_{\mathrm{w}} T_{\text {corr }}$ and $t_{1} \in\left[0, T_{\text {corr }}\right]$, namely $\left\{b_{k}(t)\right\}_{k=1}^{B_{\mathrm{w}} T_{\text {corr }}}$ are eigenfunctions of $\hat{n}(u, t)$ with same eigenvalues $2 N_{0}$. The noise for $t \in\left[0, T_{\text {corr }}\right]$ is represented as

$$
\begin{aligned}
\hat{n}\left(u, t+i T_{\mathrm{f}}\right) & =\sum_{k=1}^{B_{\mathrm{w}} T_{\text {corr }}} \hat{n}_{\mathrm{r}, i, k} b_{k}(t), \\
\hat{n}\left(u, t+i T_{\mathrm{f}}+T_{\mathrm{d}}\right) & =\sum_{k=1}^{B_{\mathrm{w}} T_{\text {corr }}} \hat{n}_{\mathrm{d}, i, k} b_{k}(t)
\end{aligned}
$$

for $i=0,1, \ldots, N_{\mathrm{s}}-1$, in which

$$
\begin{aligned}
& \hat{n}_{\mathrm{r}, i, k}=\int_{0}^{T_{\text {corr }}} \hat{n}\left(u, t+i T_{\mathrm{f}}\right) b_{k}^{*}(t) d t, \\
& \hat{n}_{\mathrm{d}, i, k}=\int_{0}^{T_{\text {corr }}} \hat{n}\left(u, t+i T_{\mathrm{f}}+T_{\mathrm{d}}\right) b_{k}^{*}(t) d t
\end{aligned}
$$

It can be computed from (4) and (7) that $\mathbb{E}\left\{\hat{n}_{\mathrm{r}, i, k}\right\}=$ $\mathbb{E}\left\{\hat{n}_{\mathrm{d}, i, k}\right\}=0, \mathbb{E}\left\{\hat{n}_{\mathrm{r}, i, k} \hat{n}_{\mathrm{r}, i, k}^{*}\right\}=\mathbb{E}\left\{\hat{n}_{\mathrm{d}, i, k} \hat{n}_{\mathrm{d}, i, k}^{*}\right\}=2 N_{0}$ for any $i, k$, and any two of $\left\{\hat{n}_{\mathrm{r}, i, k}, \hat{n}_{\mathrm{d}, i, k}\right\}_{i, k}$ are uncorrelated.

By defining

$$
\hat{\mathbf{g}} \triangleq\left[\hat{g}_{1}, \hat{g}_{2}, \ldots, \hat{g}_{B_{\mathrm{w}} T_{\text {corr }}}\right]^{\mathrm{t}},
$$

the received signal for the $0^{\text {th }}$ bit can be represented as

$$
\begin{aligned}
\hat{\mathbf{r}}=\hat{\mathbf{r}}_{\mathrm{s}}+\hat{\mathbf{n}} & =\left[\hat{\mathbf{g}}^{\mathrm{t}}, b_{0} \hat{\mathbf{g}}^{\mathrm{t}}, \hat{\mathbf{g}}^{\mathrm{t}}, b_{0} \hat{\mathbf{g}}^{\mathrm{t}}, \ldots, \hat{\mathbf{g}}^{\mathrm{t}}, b_{0} \hat{\mathbf{g}}^{\mathrm{t}}\right]^{\mathrm{t}} \\
& +\left[\hat{\mathbf{n}}_{\mathrm{r}, 0}^{\mathrm{t}}, \hat{\mathbf{n}}_{\mathrm{d}, 0}^{\mathrm{t}}, \hat{\mathbf{n}}_{\mathrm{r}, 1}^{\mathrm{t}}, \hat{\mathbf{n}}_{\mathrm{d}, 1}^{\mathrm{t}}, \ldots, \hat{\mathbf{n}}_{\mathrm{r}, N_{\mathrm{s}}-1}^{\mathrm{t}}, \hat{\mathbf{n}}_{\mathrm{d}, N_{\mathrm{s}}-1}^{\mathrm{t}}\right]^{\mathrm{t}}
\end{aligned}
$$

where

$$
\begin{aligned}
\hat{\mathbf{n}}_{\mathrm{r}, i} & =\left[\hat{n}_{\mathrm{r}, i, 1}, \hat{n}_{\mathrm{r}, i, 2}, \hat{n}_{\mathrm{r}, i, 3}, \ldots, \hat{n}_{\mathrm{r}, i, B_{\mathrm{w}} T_{\text {corr }}}\right]^{\mathrm{t}}, \\
\hat{\mathbf{n}}_{\mathrm{d}, i} & =\left[\hat{n}_{\mathrm{d}, i, 1}, \hat{n}_{\mathrm{d}, i, 2}, \hat{n}_{\mathrm{d}, i, 3}, \ldots, \hat{n}_{\mathrm{d}, i, B_{\mathrm{w}} T_{\text {corr }}}\right]^{\mathrm{t}} .
\end{aligned}
$$

By denoting

$$
\begin{gathered}
\mathbf{X}=\left[\mathbf{X}_{0}^{\mathrm{t}}, \mathbf{X}_{1}^{\mathrm{t}}, \ldots, \mathbf{X}_{N_{\mathrm{s}}-1}^{\mathrm{t}}\right]^{\mathrm{t}} \quad \text { with } \quad \mathbf{X}_{i}=\hat{\mathbf{g}}+\hat{\mathbf{n}}_{\mathrm{r}, i}, \\
\mathbf{Y}=\left[\mathbf{Y}_{0}^{\mathrm{t}}, \mathbf{Y}_{1}^{\mathrm{t}}, \ldots, \mathbf{Y}_{N_{\mathrm{s}}-1}^{\mathrm{t}}\right]^{\mathrm{t}} \quad \text { with } \quad \mathbf{Y}_{i}=b_{0} \hat{\mathbf{g}}+\hat{\mathbf{n}}_{\mathrm{d}, i},
\end{gathered}
$$

the decision rule in (3) is equivalent to

$$
D_{\mathrm{s}}=\frac{1}{2}\left(\mathbf{X}^{\mathrm{H}} \mathbf{Y}+\mathbf{Y}^{\mathrm{H}} \mathbf{X}\right) \underset{-1}{\stackrel{1}{\gtrless}} 0,
$$

where $\mathbf{X}^{\mathrm{H}}$ denotes the complex conjugate transpose of $\mathbf{X}$. Now, (12) can be equated to (9A.1) and (9A.2) in Appendix 9A in [5] by letting $A=0, B=0, C=\frac{1}{2}$ and $L=N_{\mathrm{s}} B_{\mathrm{w}} T_{\text {corr }}$. The BEP of this conventional correlation receiver is $\operatorname{Pr}\left\{D_{\mathrm{s}}<\right.$ $\left.0 \mid b_{0}=1\right\}$ due to the symmetry of the transmitted data and receiver noise, and can be computed by utilizing (9A.15) in [5]. The value of $a$ and $b$ needed in computing the BEP can be calculated using (6) as well as (9A.4) and (9A.5) in [5], which result in $a=0$ and $b=\sqrt{\frac{\eta E_{\mathrm{b}}}{N_{0}}}$ with $E_{\mathrm{b}}=2 N_{\mathrm{s}} E_{\mathrm{p}}$. The $\mathrm{BEP}$ of a conventional correlation receiver conditioned on the channel response $g(t)$ is

$$
\begin{aligned}
P_{\text {bit }}^{\mathrm{s}}=\frac{1}{2}+ & \frac{1}{2^{2 N_{\mathrm{s}} B_{\mathrm{w}} T_{\text {corr }}-1}} \sum_{l=1}^{N_{\mathrm{s}} B_{\mathrm{w}} T_{\text {corr }}}\left(\begin{array}{c}
2 N_{\mathrm{s}} B_{\mathrm{w}} T_{\text {corr }}-1 \\
N_{\mathrm{s}} B_{\mathrm{w}} T_{\text {corr }}-l
\end{array}\right) \\
\times & {\left[Q_{l}\left(0, \sqrt{\frac{\eta E_{\mathrm{b}}}{N_{0}}}\right)-Q_{l}\left(\sqrt{\frac{\eta E_{\mathrm{b}}}{N_{0}}}, 0\right)\right], }
\end{aligned}
$$


where $g(t)$ is implicitly imbedded in $E_{\mathrm{b}}$. It is obvious now that the orthogonal expansion only helps to calculate the BEP without really implementing it in the receiver. Because $Q_{l}(b, 0)=1$ for all $l, b$, and

$$
Q_{l}(0, b)=\sum_{n=0}^{l-1} \exp \left(-\frac{b^{2}}{2}\right) \frac{\left(b^{2} / 2\right)^{n}}{n !}
$$

if $l$ is an integer, (13) is further simplified to

$$
\begin{gathered}
P_{\mathrm{bit}}^{\mathrm{s}}=\frac{1}{2^{2 N_{\mathrm{s}} B_{\mathrm{w}} T_{\text {corr }}-1}} \sum_{l=1}^{N_{\mathrm{s}} B_{\mathrm{w}} T_{\text {corr }}}\left(\begin{array}{c}
2 N_{\mathrm{s}} B_{\mathrm{w}} T_{\text {corr }}-1 \\
N_{\mathrm{s}} B_{\mathrm{w}} T_{\text {corr }}-l
\end{array}\right) \\
\times \sum_{n=0}^{l-1} \frac{1}{n !} \exp \left(-\frac{\eta E_{\mathrm{b}}}{2 N_{0}}\right)\left(\frac{\eta E_{\mathrm{b}}}{2 N_{0}}\right)^{n} .
\end{gathered}
$$

One method to improve the BEP performance of the conventional correlation receiver is to average the $N_{\mathrm{s}}$ reference waveforms in one bit time to be a noise reduced correlator template. The decision statistic of this average correlation receiver is

$D_{\mathrm{a}}=\sum_{j=0}^{N_{\mathrm{s}}-1} \int_{j T_{\mathrm{f}}+T_{\mathrm{d}}}^{j T_{\mathrm{f}}+T_{\mathrm{d}}+T_{\text {corr }}} \hat{r}(t)\left[\frac{1}{N_{\mathrm{s}}} \sum_{i=-j}^{N_{\mathrm{s}}-1-j} \hat{r}\left(t+i T_{\mathrm{f}}-T_{\mathrm{d}}\right)\right] d t$ with $D_{\mathrm{a}} \underset{-1}{\stackrel{1}{\gtrless}} 0$. The BEP conditioned on each channel realization $g(t)$ can be computed by employing the same method as for the conventional correlation receiver, but now $\mathbf{X}=$ $\sum_{i=0}^{N_{\mathrm{s}}-1} \mathbf{X}_{i}$ and $\mathbf{Y}=\frac{1}{N_{\mathrm{s}}} \sum_{i=0}^{N_{\mathrm{s}}-1} \mathbf{Y}_{i}$ instead of the definitions in (10) and (11). Equation (9A.1) and (9A.2) in [5] are equated to $D_{\mathrm{a}}$ by letting $A=0, B=0, C=\frac{1}{2}$ and $L=B_{\mathrm{w}} T_{\text {corr }}$. The BEP of this average correlation receiver conditioned on the channel realization $g(t)$ by substituting $a=0$ and $b=\sqrt{\frac{\eta E_{\mathrm{b}}}{N_{0}}}$ into (9A.15) in [5] after simplification is

$$
\begin{gathered}
P_{\text {bit }}^{\mathrm{a}}=\frac{1}{2^{2 B_{\mathrm{w}} T_{\text {corr }}-1}} \sum_{l=1}^{B_{\mathrm{w}} T_{\text {corr }}}\left(\begin{array}{c}
2 B_{\mathrm{w}} T_{\text {corr }}-1 \\
B_{\mathrm{w}} T_{\text {corr }}-l
\end{array}\right) \\
\times \sum_{n=0}^{l-1} \frac{1}{n !} \exp \left(-\frac{\eta E_{\mathrm{b}}}{2 N_{0}}\right)\left(\frac{\eta E_{\mathrm{b}}}{2 N_{0}}\right)^{n} .
\end{gathered}
$$

The average BEP of the conventional and average correlation receivers over channels can be obtained if $\mathbb{E}\left\{\exp \left(-\frac{\eta E_{\mathrm{b}}}{2 N_{0}}\right)\left(\frac{\eta E_{\mathrm{b}}}{2 N_{0}}\right)^{n}\right\}$ exits, i.e., the moment generating function of $\frac{\eta E_{\mathrm{b}}}{2 N_{0}}$ exists.

\section{INTEGRATION TIME ANALYSIS}

The integration time of the correlator $T_{\text {corr }}$ affects the BEP which can be seen in (14) and (15). Conditioned on a channel realization, the efficiency factor $\eta$ increases as $T_{\text {corr }}$ increases, therefore $\exp \left(-\eta E_{\mathrm{b}} / 2 N_{0}\right)$ decreases but $\left(\eta E_{\mathrm{b}} / 2 N_{0}\right)^{n}$ increases. In addition, the number of terms in the summation also increases as $T_{\text {corr }}$ increases. Thus the BEP has its minimum at some value of $T_{\text {corr }}$, and starts to raise as $T_{\text {corr }}$ diverges from this value. In the following, the optimal $T_{\text {corr }}$ of a conventional correlation receiver is discussed, but can be easily generalized to different kinds of correlation receivers.
Due to the simple receiver constraint and that it is difficult to implement an adaptive algorithm using analog devices, the value of $T_{\text {corr }}$ is fixed once the receiver is implemented. The best choice of $T_{\text {corr }}$ is to minimize the average BEP which is immediately seen a difficult task from (14) because frequency selective UWB channels with random path arrival times make finding the distribution of $\frac{\eta E_{\mathrm{b}}}{2 N_{0}}$ difficult. By defining the decision SNR

$$
f_{\mathrm{s}}\left(T_{\text {corr }}\right)=\frac{N_{\mathrm{s}}\left(\eta E_{\mathrm{p}}\right)^{2}}{N_{0} \eta E_{\mathrm{p}}+\frac{B_{\mathrm{w}} T_{\text {corr }} N_{0}^{2}}{2}},
$$

which is the ratio of the signal energy to the noise power in the decision statistic $D_{\mathrm{s}}$, another choice to optimize the integration time is to maximize $f_{\mathrm{s}}\left(T_{\text {corr }}\right)$. This criterion is equivalent to minimizing $N_{\mathrm{s}} f_{\mathrm{s}}^{-1}\left(T_{\text {corr }}\right)$

$$
N_{\mathrm{s}} f_{\mathrm{s}}^{-1}\left(T_{\text {corr }}\right)=\frac{N_{0}}{\eta E_{\mathrm{p}}}+\frac{B_{\mathrm{w}} T_{\text {corr }}}{2}\left(\frac{N_{0}}{\eta E_{\mathrm{p}}}\right)^{2},
$$

which indicates that the optimal integration time based on this criterion depends on the energy per pulse $E_{\mathrm{p}}$ instead of energy per bit $E_{\mathrm{b}}$. Minimizing $N_{\mathrm{s}} f_{\mathrm{s}}^{-1}\left(T_{\text {corr }}\right)$ is still difficult to manage theoretically. By exploiting the average power profile of the received signal which is assumed exponential decays here without loss of generality [6], then

$$
\mathbb{E}\left\{g^{2}(t)\right\}=\Omega a \exp (-a t)
$$

where $\Omega=\mathbb{E}\left\{E_{\mathrm{p}}\right\}$ and $\frac{1}{a}$ is the power decay time constant. Under the exponential power decay profile assumption,

$$
\mathbb{E}\left\{\eta E_{\mathrm{p}}\right\}=\Omega\left[1-\exp \left(-a T_{\text {corr }}\right)\right] .
$$

In the following three subsections, we are going to replace $\eta E_{\mathrm{p}}$ in $f_{\mathrm{s}}\left(T_{\text {corr }}\right)$ by $\mathbb{E}\left\{\eta E_{\mathrm{p}}\right\}$, and investigate the effects of choosing different values of $T_{\text {corr }}$. Note that $f_{\mathrm{s}}\left(T_{\text {corr }}\right)$ with this substitution does not equal the average decision SNR over channel statistics, and the observations we obtain will be justified in the next section by evaluating the average decision SNR and average BEP numerically.

\section{A. Minimal integration time}

A special case in which the noise power is extremely large is considered. Under this condition,

$$
\left(\frac{N_{0}}{\eta E_{\mathrm{p}}}\right)^{2} \gg \frac{N_{0}}{\eta E_{\mathrm{p}}},
$$

and the quantity we want to minimize is approximate

$$
N_{\mathrm{s}} f_{\mathrm{s}}^{-1}\left(T_{\text {corr }}\right) \cong \frac{B_{\mathrm{w}} T_{\text {corr }}}{2}\left(\frac{N_{0}}{\eta E_{\mathrm{p}}}\right)^{2} .
$$

By replacing $\eta E_{\mathrm{p}}$ with $\mathbb{E}\left\{\eta E_{\mathrm{p}}\right\}$, we now want to minimize

$$
\frac{B_{\mathrm{w}} T_{\text {corr }}}{2} \times\left(\frac{N_{0}}{\Omega}\right)^{2} \times\left[\frac{1}{1-\exp \left(-a T_{\text {corr }}\right)}\right]^{2},
$$

which is a convex function of $T_{\text {corr }} \in(0, \infty)$, and has an unique minimum. After differentiating (19) with respect to $T_{\text {corr }}$ and equating it 0 , the equation which determines the optimal value of $T_{\text {corr }}$ is

$$
\ln \left(1+2 a T_{\text {corr }}\right)=a T_{\text {corr }},
$$


which does not depend on the receiver bandwidth $B_{\mathrm{w}}$ and $\Omega / N_{0}$. In this extremely high noise power case, the value of $T_{\text {corr }}$ only depends on the power decay time constant $\frac{1}{a}$. The solution of (20) is $a T_{\text {corr }}=1.2564$, and

$$
T_{\text {corr }}=\frac{1.2564}{a}=1.2564 \times \text { time constant. }
$$

This high noise power case represents the minimum value of $T_{\text {corr }}$ for a conventional correlation receiver, and $T_{\text {corr }}$ in a general situation should be larger than this value. For another special case that the received signal power is extremely high, it is not really meaningful because the integration time then should be as long as possible, i.e., the channel delay spread.

\section{B. Optimal integration time}

For the normal $E_{\mathrm{p}} / N_{0}$ case, $\eta E_{\mathrm{p}}$ in (16) is replaced by (17), and the quantity to be minimized is

$$
\begin{aligned}
N_{\mathrm{s}} f_{\mathrm{s}}^{-1}\left(T_{\text {corr }}\right) & =\frac{N_{0}}{\Omega} \times \frac{1}{1-\exp \left(-a T_{\text {corr }}\right)} \\
& +\frac{B_{\mathrm{w}} T_{\text {corr }}}{2}\left[\frac{N_{0}}{\Omega} \times \frac{1}{1-\exp \left(-a T_{\text {corr }}\right)}\right]^{2} .
\end{aligned}
$$

The right hand side of (22) is differentiated with respect to $T_{\text {corr }}$ to achieve

$$
\begin{gathered}
\left(\frac{2 \Omega}{B_{\mathrm{w}} N_{0}}+1+2 a T_{\text {corr }}\right) \exp \left(-a T_{\text {corr }}\right) \\
-\frac{2 \Omega}{B_{\mathrm{w}} N_{0}} \exp \left(-2 a T_{\text {corr }}\right)-1=0 .
\end{gathered}
$$

Equation (23) shows that the optimal integration time depends on $B_{\mathrm{w}}, 1 / a$, and $\Omega / N_{0}$. Given $B_{\mathrm{w}}$ and $\Omega / N_{0}$, the value of $a T_{\text {corr }}$ which makes (23) sustained can be computed numerically. For a specific BEP, the required pulse energy increases and the optimal integration time decreases as $B_{\mathrm{w}}$ increases because of the increasing incoming noise.

\section{Performance degradation V.S. excess or lack of integration}

The optimal integration time changes according to application environments, but the value adopted by the receiver is difficult to change once the correlator is implemented. How much the performance degrades because of the excessive or short integration should be considered before choosing the adequate value. In (16) with $\eta E_{\mathrm{p}}$ replaced by $\mathbb{E}\left\{\eta E_{\mathrm{p}}\right\}$, $N_{\mathrm{s}} f_{\mathrm{s}}^{-1}\left(T_{\text {corr }}\right)$ includes two portions

$$
\begin{aligned}
g_{\mathrm{s}}\left(T_{\text {corr }}\right) & =\frac{N_{0}}{\Omega} \times \frac{1}{1-\exp \left(-a T_{\text {corr }}\right)}, \\
h_{\mathrm{s}}\left(T_{\text {corr }}\right) & =\frac{B_{\mathrm{w}} T_{\text {corr }}}{2}\left[\frac{N_{0}}{\Omega} \times \frac{1}{1-\exp \left(-a T_{\text {corr }}\right)}\right]^{2} .
\end{aligned}
$$

Equation (24) indicates that $g_{\mathrm{s}}\left(T_{\text {corr }}\right)$ decreases as $T_{\text {corr }}$ increases for $T_{\text {corr }} \in[0, \infty)$. Equation (25) shows that $h_{\mathrm{s}}\left(T_{\text {corr }}\right)$ also decreases as $T_{\text {corr }}$ increases for $T_{\text {corr }} \in\left[0, t_{\mathrm{B}}\right)$ with some value $t_{\mathrm{B}}$, then starts to increase as $T_{\text {corr }}$ increases for $T_{\text {corr }} \geq t_{\mathrm{B}}$. The value of $t_{\mathrm{B}}$ is determined by $B_{\mathrm{w}}, N_{0} / \Omega$ and $1 / a$, and the optimal $T_{\text {corr }}$ is greater than or equal to $t_{\mathrm{B}}$. Another observation from (24) and (25) is that $g_{\mathrm{s}}\left(T_{\text {corr }}\right)$ for $T_{\text {corr }} \in(0, \infty)$ as well as $h_{\mathrm{s}}\left(T_{\text {corr }}\right)$ for $T_{\text {corr }} \in\left(0, t_{\mathrm{B}}\right)$ decrease roughly exponentially

\begin{tabular}{|l|l|l|l|l|}
\hline & $\mathrm{cm} 1$ & $\mathrm{~cm} 2$ & $\mathrm{~cm} 3$ & $\mathrm{~cm} 4$ \\
\hline$\frac{1}{a}(\mathrm{~ns})$ & 4 & 8 & 12 & 16 \\
\hline$\lambda(1 / \mathrm{ns})$ & 3.5 & 3.5 & 3.5 & 3.5 \\
\hline$\eta:$ mean & 0.85908 & 0.83218 & 0.82051 & 0.81577 \\
\hline$\eta:$ std & 0.0432 & 0.041828 & 0.039349 & 0.038193 \\
\hline
\end{tabular}

TABLE I

CHANNEL PARAMETERS AND THE EFFICIENCY FACTOR $\eta$.
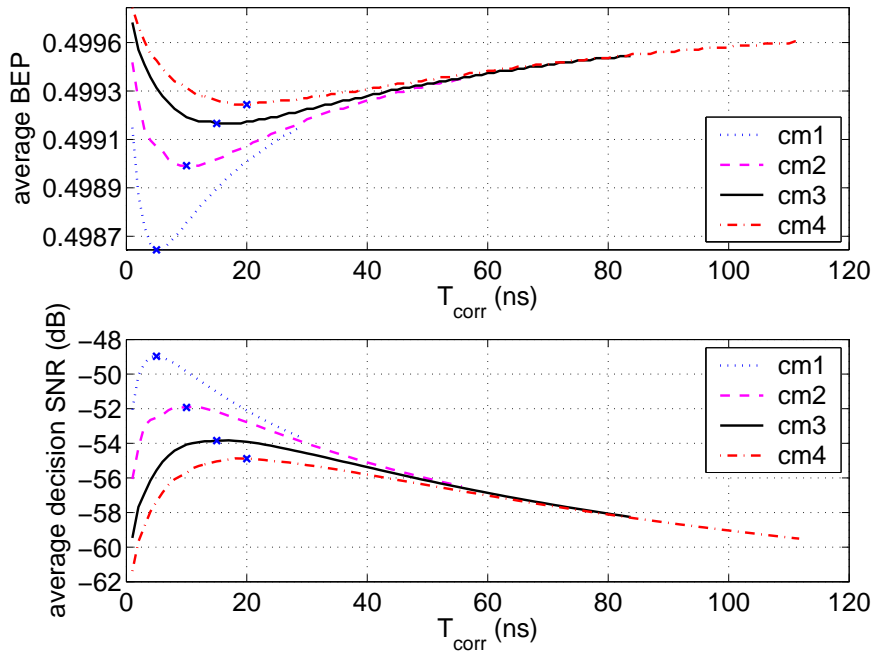

Fig. 1. Average BEP and average decision SNR for $E_{\mathrm{p}} / N_{0}=-23 \mathrm{~dB}$ $\left(E_{\mathrm{b}} / N_{0}=-10 \mathrm{~dB}\right)$ with $N_{\mathrm{s}}=10$.

as $T_{\text {corr }}$ increases, and $h_{\mathrm{s}}\left(T_{\text {corr }}\right)$ for $T_{\text {corr }} \in\left[t_{\mathrm{B}}, \infty\right)$ increases roughly linearly. Therefore, under integration degrades BEP performance more than over integration.

\section{NUMERICAL EXAMPLES}

This section uses a channel model in (26) to analyze the average BEP and average decision SNR versus $T_{\text {corr }}$ numerically to verify the analysis and observations in Section III. The model is

$$
h(t)=\sum_{l=0}^{L} \alpha_{l} \delta\left(t-T_{l}\right),
$$

where $\alpha_{l}$ and $T_{l}$ are the amplitude and arrival time of the $l^{\text {th }}$ path. The magnitude of $\alpha_{l}$ has lognormal distribution, and the polarity of it can be +1 or -1 with equal probability. In addition, $\alpha_{l}$ and $\alpha_{j}$ are independent for $l \neq j$. The energy of a single transmitted pulse is normalized to 1 , and $\mathbb{E}\left\{\alpha_{l}^{2}\right\}=c \exp \left(-a T_{l}\right)$ with some constant $c$ such that $\sum_{l} \mathbb{E}\left\{\alpha_{l}^{2}\right\}=E_{\mathrm{p}}$. The channel delay spread $T_{\mathrm{mds}}$ is defined as the interval containing $99 \%$ of the energy in the average received waveform. The probability that a path arrives at time $T_{l}$ has poisson distribution with the path arrival rate $\lambda$. The receiver bandwidth is equal to $4 \mathrm{GHz}$, and 100 channel realizations are generated to get the numerically average BEP and decision SNR. The parameters used in this numerical analysis are listed in Table I. The resolution of searching the optimal $T_{\text {corr }}$ is equal to $1 \mathrm{~ns}$. 
Figure 1 shows the average decision SNR and BEP for the extremely large noise power case. Crosses in the figure, which mark the positions of the optimal integration time $\left(T_{\mathrm{corr}}^{\mathrm{opt}}\right)$ for each channel model, indicate that $T_{\text {corr }}^{\mathrm{opt}}$ acquired by using these two criteria are the same and fit the results predicted by (21). This figure also shows that for a fixed $N_{\mathrm{s}}, B_{\mathrm{w}}$ and $E_{\mathrm{b}} / N_{0}$, the value of $T_{\text {corr }}^{\mathrm{opt}}$ increases as $\frac{1}{a}$ increases but with worse performance because the incoming noise power also increases. This figure verifies that excessive integration harms the performance less than short integration.

Figure 2 and 3 show $T_{\text {corr }}^{\mathrm{opt}}$ acquired through minimizing the average BEP, maximizing the average decision SNR, and fining the solution of (23), as well as the corresponding performance. In Figure 2, the values of $T_{\text {corr }}^{\mathrm{opt}}$ obtained through different criteria are close at small $E_{\mathrm{f}} / N_{0}$, but could be different at large $E_{\mathrm{f}} / N_{0}$. Minimizing the average BEP produces larger $T_{\mathrm{corr}}^{\mathrm{opt}}$ than maximizing the average decision SNR, and the value of $T_{\text {corr }}^{\mathrm{opt}}$ increases as $E_{\mathrm{f}} / N_{0}$ increases. The value of $T_{\text {corr }}^{\mathrm{opt}}$ obtained by solving (23) is the largest one among the three because the received waveform energy acquired by integrating an exponential function can be overestimated. Even divergence resulted from different criteria is demonstrated, Figure 2 and 3 display that the influence of this divergence on both the average BEP and the average decision SNR is small, which allows us to acquire $T_{\text {corr }}^{\mathrm{opt}}$ easily through solving (23) or maximizing the average decision SNR instead of minimizing the average BEP. Figure 2 also shows that compared to integrating over the channel delay spread, the correlator adopting the optimal integration time can have approximate $2 \mathrm{~dB}$ gain at $\mathrm{BEP}=1 \mathrm{e}-4$. As $E_{\mathrm{f}} / N_{0}$ increases, $T_{\text {corr }}^{\mathrm{opt}}$ approaches $T_{\mathrm{mds}}$. Table I includes the mean and the standard deviation of the efficiency factor $\eta$ over the 100 channel realizations with the optimal integration time for the average $\mathrm{BEP}=1 \mathrm{e}-4$ and $N_{\mathrm{s}}=10$. The mean value of $\eta$ decreases as $\frac{1}{a}$ increases, and small standard deviations show that the value of $\eta$ for every channel realization is close to each other.

\section{CONCLUSiOnS}

The BEP of UWB conventional and average correlation receivers are analyzed with the help of the orthogonal expansion theorem. The optimal integration time of a UWB conventional correlation receiver is also analyzed. It shows that the integration time has a minimal value which is not related to the receiver bandwidth and the bit repetition time. This minimal value corresponds to an extremely small SNR case, and the real operational value should be greater than this one. It also shows that excessive integration harms the performance less than short integration. With the parameters used in those numerical examples, about $2 \mathrm{~dB}$ gain can be get by using optimal integration time instead of channel delay spread in the correlator in the interested BEP range.

\section{REFERENCES}

[1] M. Z. Win, R. A. Scholtz, "On the robustness of ultra-wide bandwidth signals in dense multipath environments", IEEE Commun. Lett., vol. 2, pp. 51-53, Feb. 1998.

[2] M. Z. Win, R. A. Scholtz, "On the energy capture of ultra-wide bandwidth signals in dense multipath environments", IEEE Commun. Lett., vol. 2, Sep. 1998, pp. 245-247.
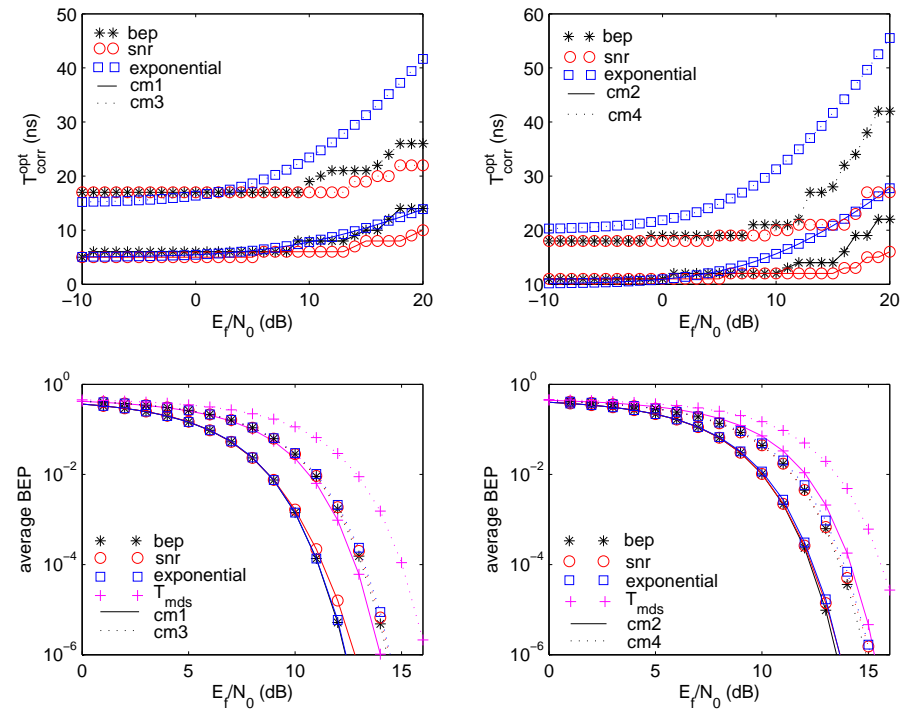

Fig. 2. The optimal integration time obtained by minimizing the average BEP (labelled bep), maximizing the average decision SNR (labelled snr), and solving (23) (labelled exponential) with $N_{\mathrm{s}}=10$, as well as the corresponding average BEP.
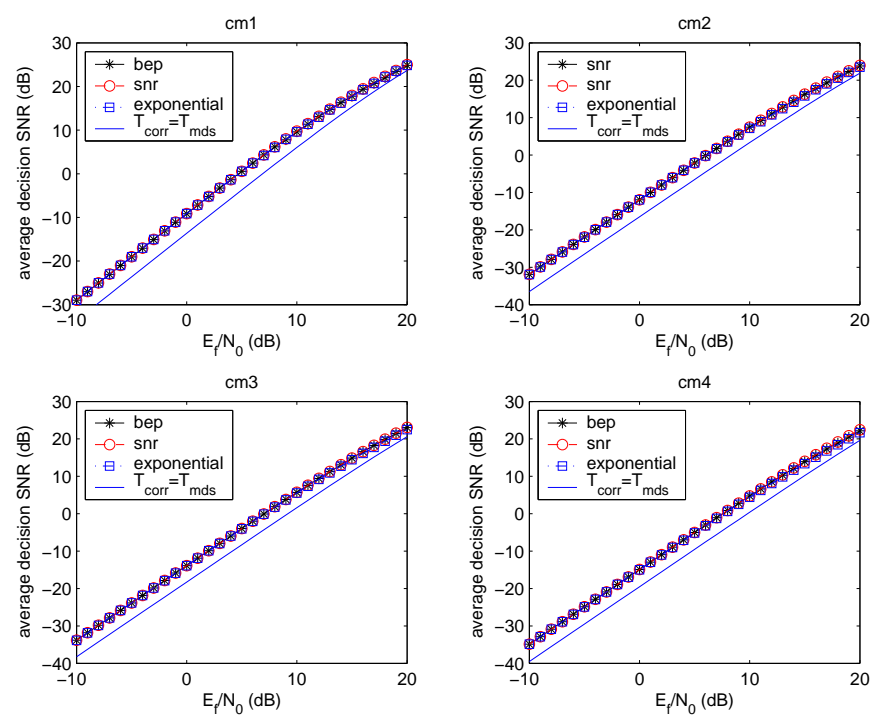

Fig. 3. The average decision SNR with optimal integration time obtained by minimizing the average BEP (labelled bep), maximizing the average decision SNR (labelled snr), and solving (23) (labelled exponential) with $N_{\mathrm{s}}=10$.

[3] R. T. Hoctor and H. W. Tomlinson, "An overview of delay-hopped transmitted-reference RF communications", Technique Information Series: G.E. Research and Development Center, January 2002.

[4] Y.-L. Chao and R. A. Scholtz, "Optimal and Suboptimal Receivers for Ultra-wideband Transmitted Reference Systems", Globcom, December, 2003.

[5] M. K. Simon and M.-S. Alouini, Digital Communication over Fading Channels: A Unified Approach to Performance Analysis, John Wiley and Sons, Inc., 2000.

[6] M. A. Nemati and R. A. Scholtz, "A Diffusion Model for UWB Indoor Propagation", Milcom, 2004. 